Supporting Information for:

\title{
Surface-Directed Assembly of Sequence-Defined Synthetic Polymers into Networks of Hexagonally-Patterned Nanoribbons with Controlled Functionalities
}

\author{
Chun-Long Chen, ${ }^{*},+*$ Ronald N. Zuckermann ${ }^{\ddagger}$ and James J. DeYoreo ${ }^{\dagger,+, \S}$ \\ ${ }^{\dagger}$ Physical Sciences Division, Pacific Northwest National Laboratory, Richland, WA 99352, \\ ॠ Molecular Foundry, Lawrence Berkeley National Laboratory, Berkeley, CA 94720 \\ ${ }^{\S}$ Departments of Materials Science and Engineering and of Chemistry, University of Washington, Seattle, \\ WA 98195 \\ *Corresponding author. E-mail: $\underline{\text { Chunlong.Chen@pnnl.gov }}$

\section{Content} \\ S1. Methods \\ S2. Supporting Figures \\ S3. References
}




\section{S1. Methods}

\section{Peptoid Synthesis}

All peptoids and peptoid-peptide hybrids were synthesized on a commercial Aapptec Apex 396 robotic synthesizer on using a solid-phase submonomer cycle as described previously. ${ }^{1,2}$ All amine submonomers and other reagents used for our peptoid synthesis are obtained from commercial sources and used without further purification. Rink amide resin $(0.60 \mathrm{mmol} / \mathrm{g}$, Novabiochem, Cat No. 01-64-0013) was used to generate C-terminal amide peptoids. In this method, the Fmoc group on the resin was deprotected by adding $2 \mathrm{~mL}$ of $20 \%(\mathrm{v} / \mathrm{v})$ 4-Methylpiperidine/N,N-dimethylformamide (DMF), agitating for $20 \mathrm{~min}$, draining, and washing with DMF. All DMF washes consisted of the addition of $1 \mathrm{~mL}$ of DMF, followed by agitation for $1 \mathrm{~min}$ (repeated five times). An acylation reaction was then performed on the amino resin by the addition of $1.0 \mathrm{~mL}$ of $1.2 \mathrm{M}$ bromoacetic acid in DMF, followed by $0.18 \mathrm{~mL}$ of $\mathrm{N}, \mathrm{N}-$ diisopropylcarbodiimide (DIC, $1.15 \mathrm{mmol}$, neat). The mixture was agitated for $20 \mathrm{~min}$ at room temperature, drained, and washed with DMF. Nucleophilic displacement of the bromide with various primary amines occurred by a $1.0 \mathrm{~mL}$ addition of the primary amine monomer as a $1.0-1.5 \mathrm{M}$ solution in $\mathrm{N}$-methyl-2-pyrrolidone (NMP), followed by agitation for $60 \mathrm{~min}$ at room temperature. The monomer solution was drained from the resin, and the resin was washed with DMF as described above. The acylation and displacement steps were repeated until a polypeptoid of the desired length was synthesized. All reactions were performed at room temperature. The resultant crudes were cleaved from the resin by addition of $4.0 \mathrm{~mL} \mathrm{95 \%} \mathrm{(v/v)} \mathrm{trifluoroacetic} \mathrm{acid} \mathrm{(TFA)} \mathrm{in} \mathrm{water} \mathrm{for} 20 \mathrm{~min}$. Following cleavage, the crudes were dissolved in $4.0 \mathrm{~mL}$ mixture $(\mathrm{v} / \mathrm{v}=1: 1)$ of water and acetonitrile for further purification.

For the synthesis of EEEG-Pep-1 and DGRG-Pep-1, amino acids such as glycine, aspartic acid, glutamic aicd and arginine were incorporated into the peptoid by using a standard peptide synthesis method. FmocGly-OH (Novabiochem), Fmoc-Asp, Fmoc-Glu and Fmoc-Arg(Pbf)-OH (Novabiochem) in N-methyl-2pyrrolidone (NMP) $(0.8 \mathrm{mmol}$ in $2 \mathrm{~mL}$ of NMP) was added to the resin-bound amine with $0.4 \mathrm{M}$ hydroxybenzotriazole in DMF and $137 \mu \mathrm{L}$ of $N, N^{\prime}$-diisopropylcarbodiimide (DIC) $(0.92 \mathrm{mmol})$. The reaction mixture was agitated for 2 hours. When the Fmoc-Gly-OH was added next to a peptoid backbone, the reaction mixture was added twice for efficient coupling. Fmoc group was then deprotected with $20 \%$ piperidine in DMF for further synthesis. For these two functionalized peptoids, they were cleaved from resins using the mixture of 92.5:5:2.5 TFA/water/triethylsilane (v/v/v).

All peptoids and peptoid-peptide hybrids were purified by reverse-phase HPLC on a Vydac C4 or C18 column $(10 \mu \mathrm{m}, 22 \mathrm{~mm} \times 250 \mathrm{~mm})$, using a gradient of 5-95\% acetonitrile in water with $0.1 \%$ TFA over 60 min. All final products were analyzed by analytical reverse-phase HPLC (5 - 95\% gradient at 1.0 $\mathrm{mL} / \mathrm{min}$ over 30 minutes at $60^{\circ} \mathrm{C}$ with a Vydac C18, $5 \mu \mathrm{m}, 4.6 \times 150 \mathrm{~mm}$ column), and electrospray mass spectrometry (Agilent 1100 series LC/MSD trap system, Agilent Technologies, Santa Clara,CA) or matrix-assisted laser desorption/ionization mass spectrometry (Applied Biosytem/MDS SCIEX 4800 MALDI TOF/TOF Analyzer). The final peptoid or peptoid-peptide hybrid products were lyophilized at least twice from their solution in mixture $(\mathrm{v} / \mathrm{v}=1: 1)$ of water and acetonitrile. All lyophilized products were finally divided into small portions $\left(3.0 \times 10^{-6} \mathrm{~mol}\right)$ and stored at $-80^{\circ} \mathrm{C}$.

For relative hydrophobicity test: purified peptoids were analyzed using Waters ACQUITY reverse-phase UPLC $\left(5-95 \% \mathrm{CH}_{3} \mathrm{CN}\right.$ in $\mathrm{H}_{2} \mathrm{O}$ at $0.4 \mathrm{~mL} / \mathrm{min}$ over $5 \mathrm{~min}$ at $40^{\circ} \mathrm{C}$ with a ACQUITY ${ }^{\circledR B E H ~} \mathrm{C} 18,1.7 \mu \mathrm{m}$, $2.1 \mathrm{~mm} \times 50 \mathrm{~mm}$ column) that was connected with a Waters SQD2 mass spectrometry system.

\section{Peptoid sequences and their UPLC-MS characterizations}

Structures of the synthesized peptoids and molecular weight of each peptoid as determined by mass spectrometry are shown below. 
Pep-1: 1965.6 (Molecular weight), 1967.0 (Found:[M+H] $]^{+}$), 983.9 (Found:[M/2+H] $]^{+}$).
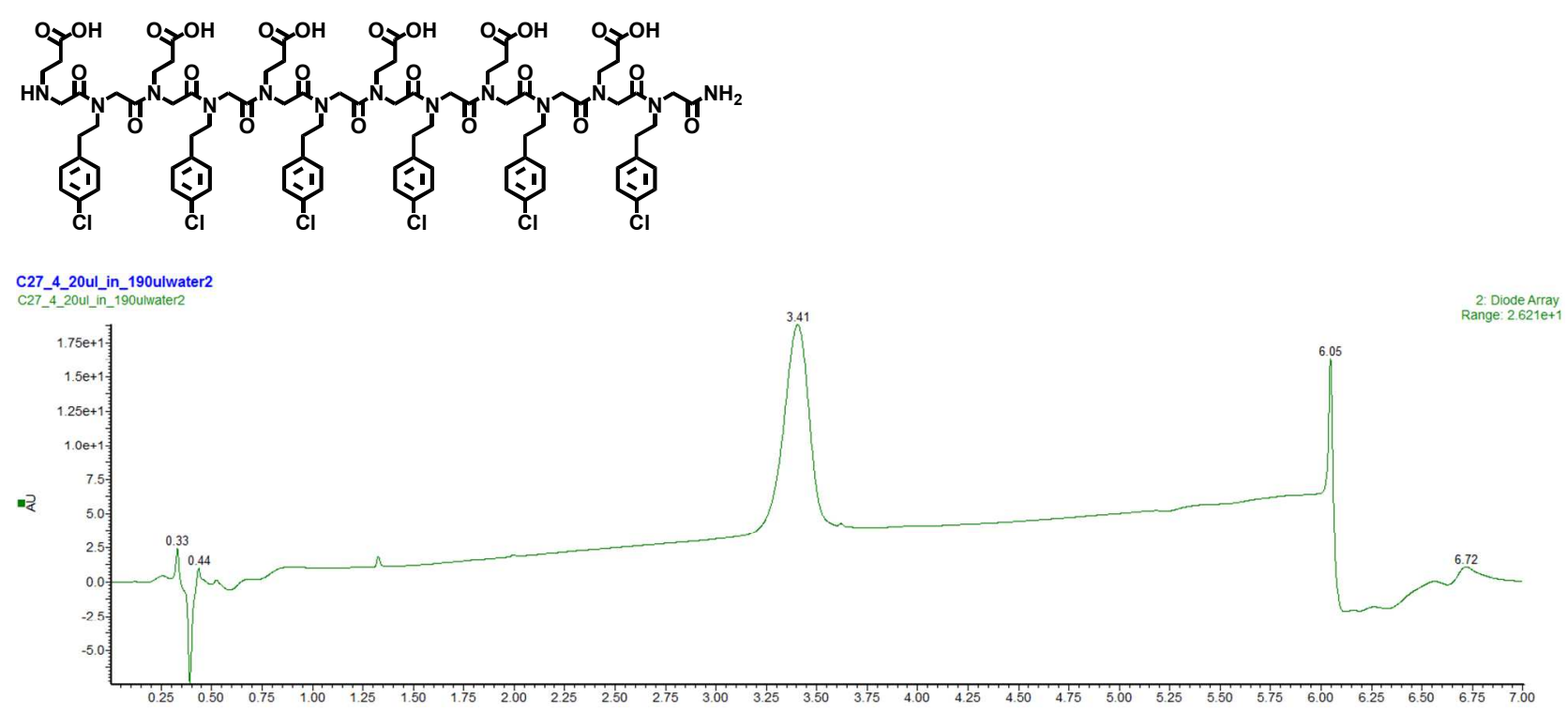

UPLC characterization of Pep-1

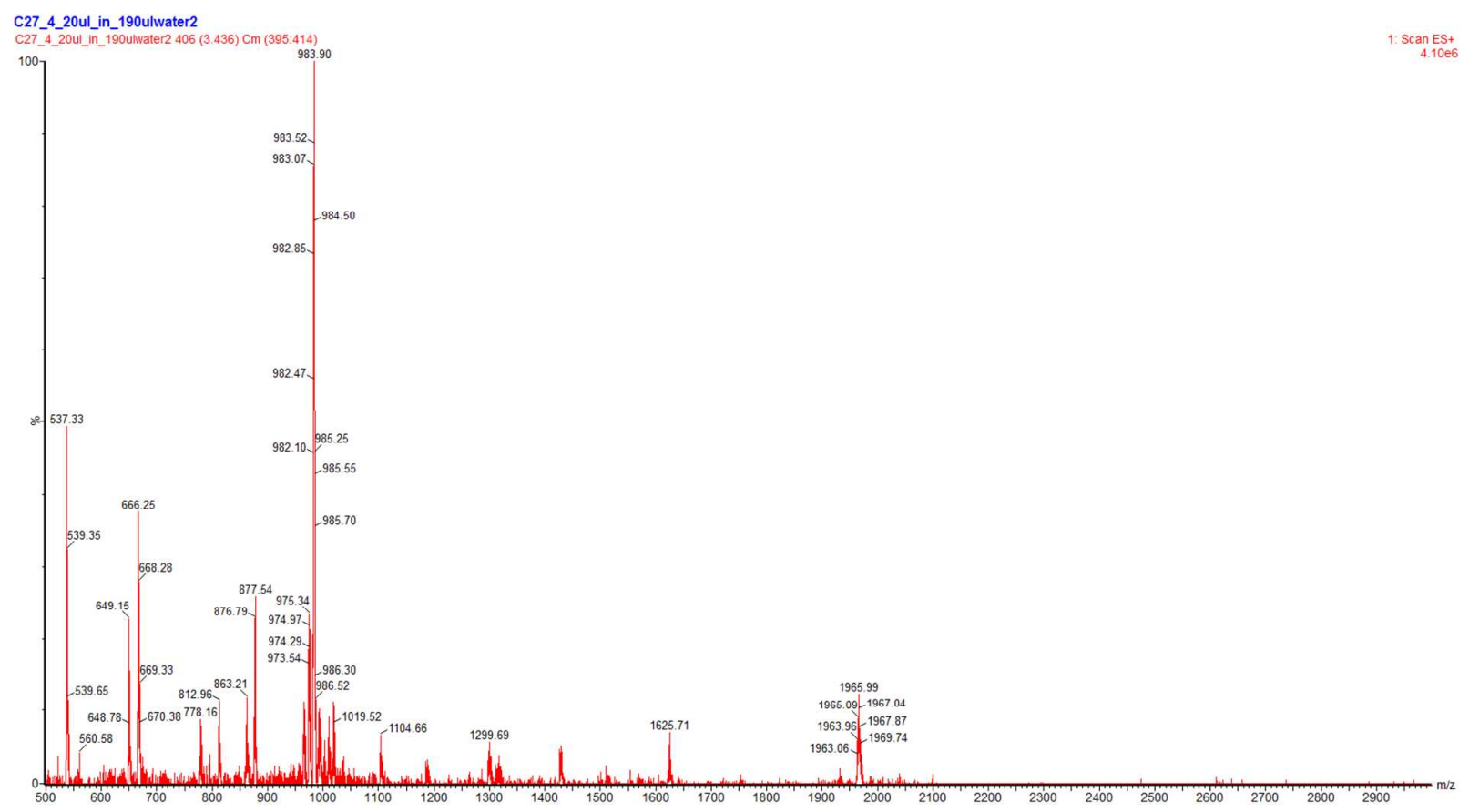

MS characterization of Pep-1 
Pep-2: 2068.9 (Molecular weight), 2070.9 (Found:[M+H $]^{+}$), 1035.4 (Found:[M/2+H] $]^{+}$).
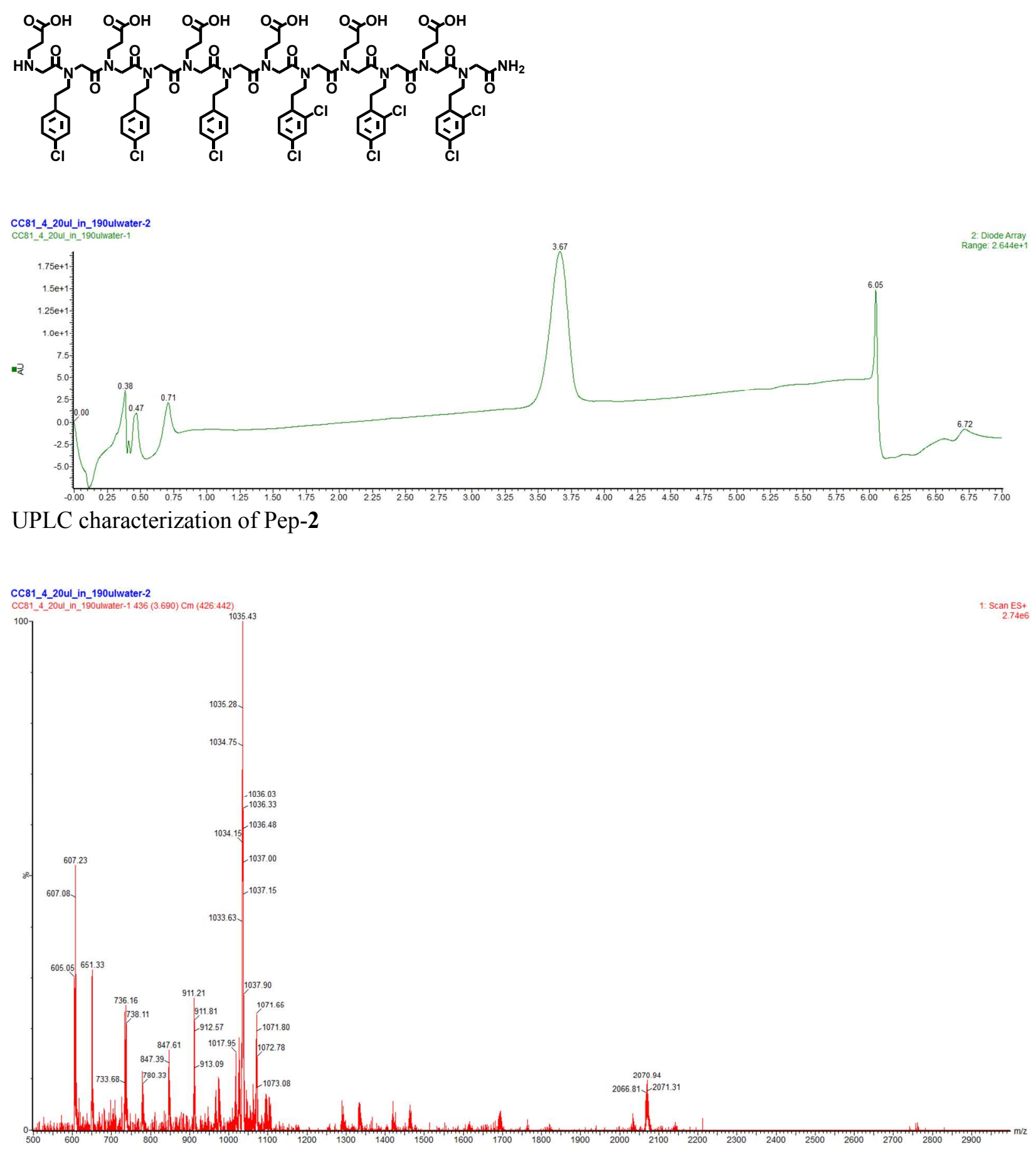

MS characterization of Pep-2 
Pep-3: 1896.7 (Molecular weight), 1897.9 (Found:[M+H] $]^{+}$), 949.5 (Found:[M/2+H] $]^{+}$).
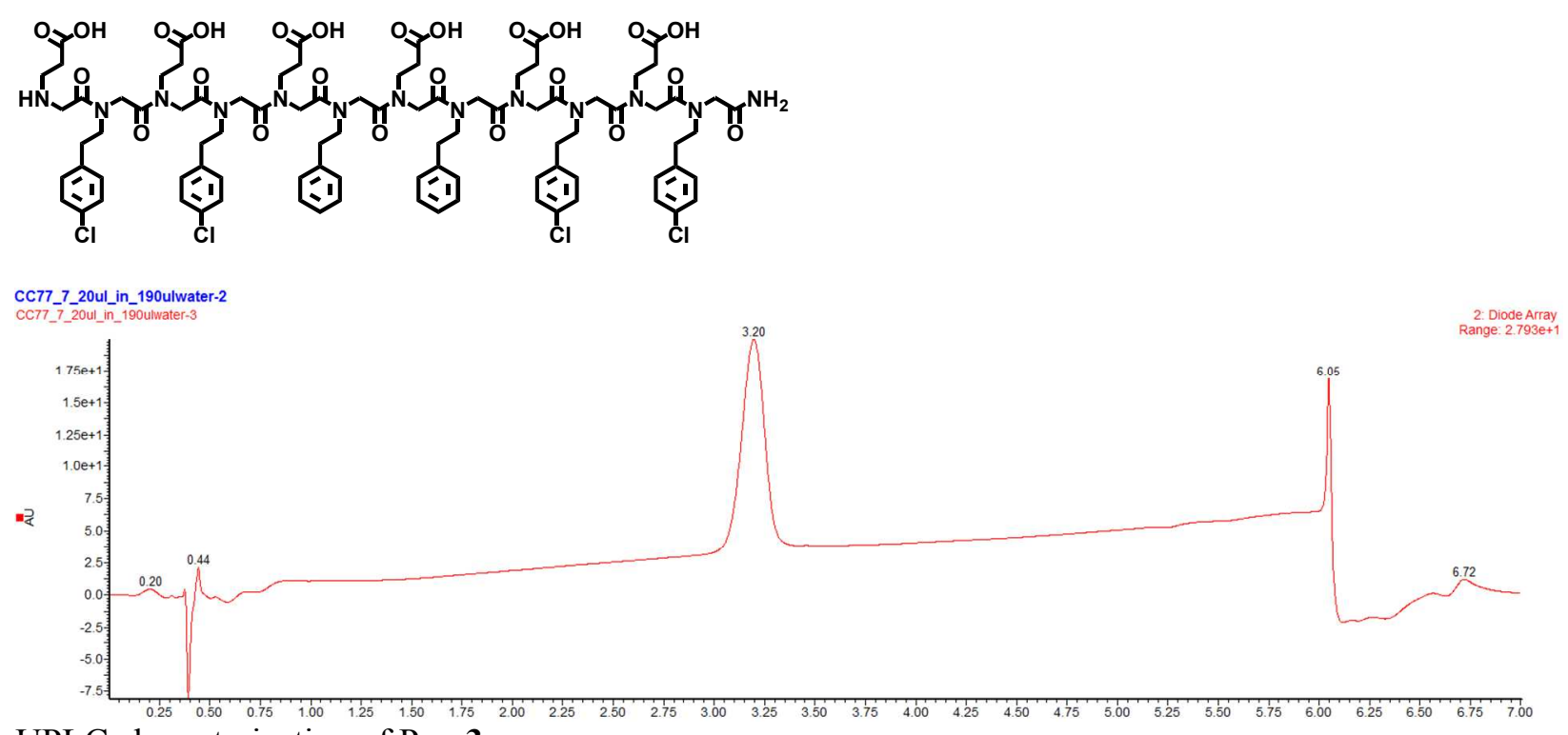

UPLC characterization of Pep-3

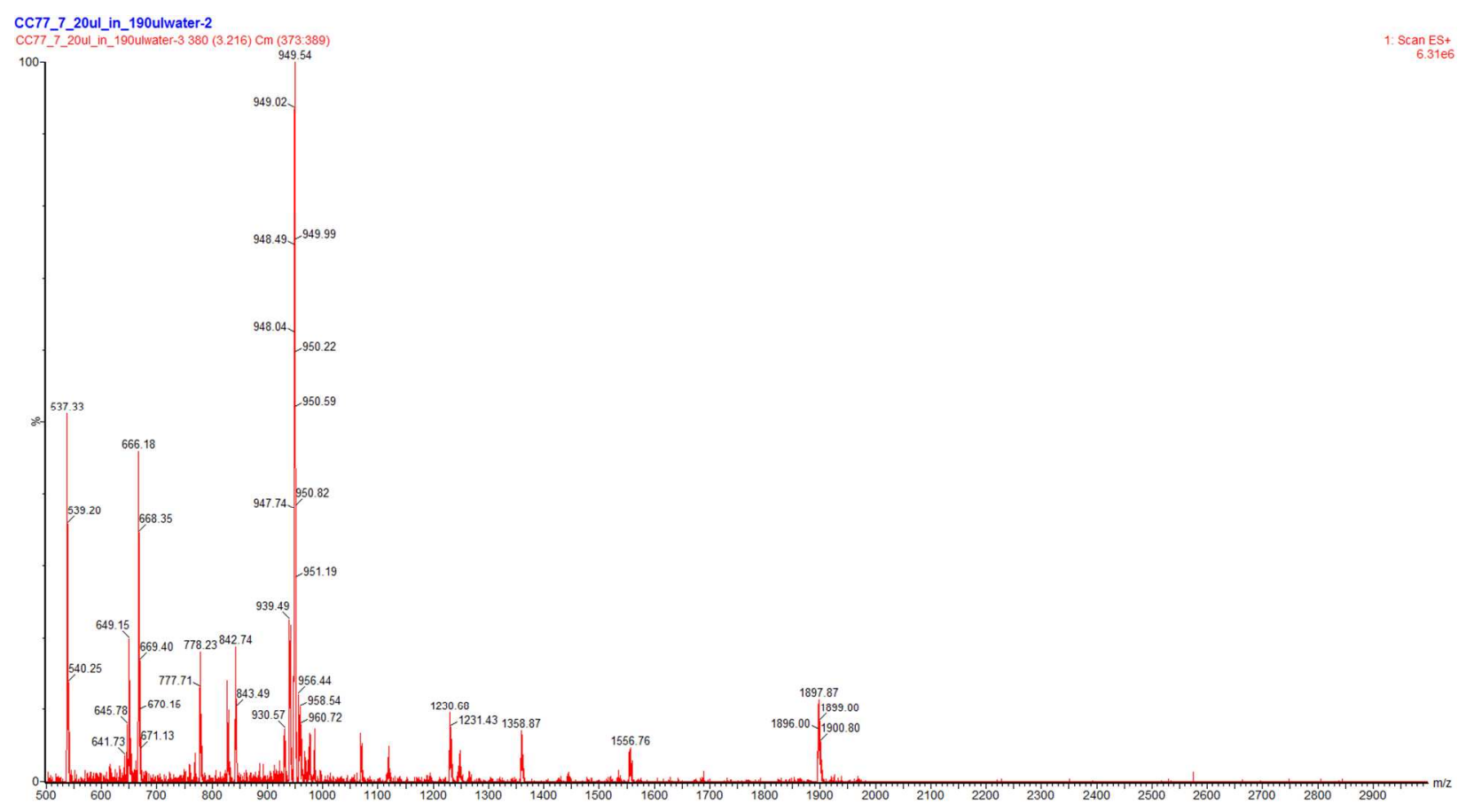

MS characterization of Pep-3 
Pep-4: 1896.7 (Molecular weight), 1898.0 (Found:[M+H] $]^{+}$), 948.9 (Found:[M/2+H] $]^{+}$).
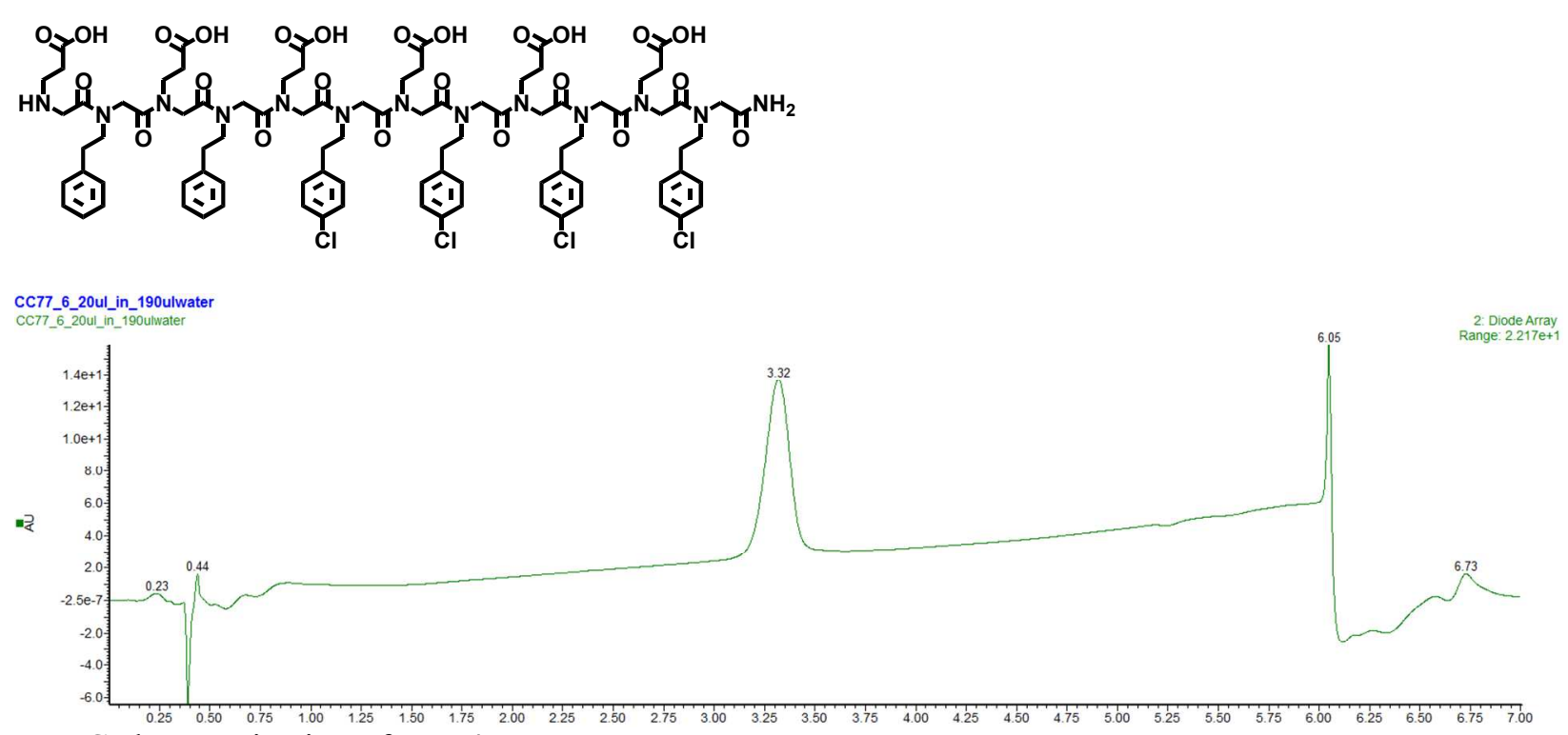

UPLC characterization of Pep-4

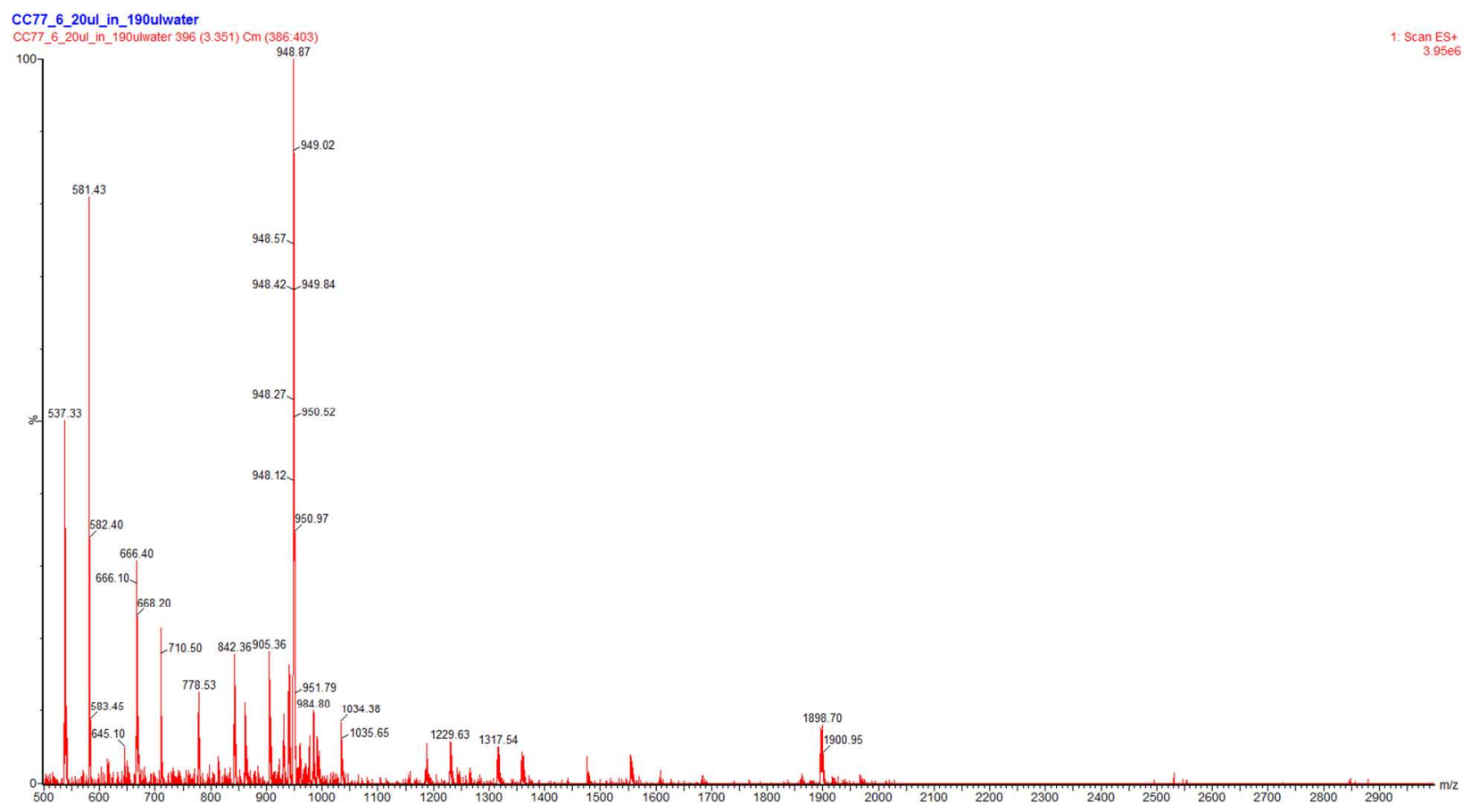

MS characterization of Pep-4 
EEEG-Pep-1: 2410.0 (Molecular weight), (Found:[M+H] $]^{+}$), 1206.2 (Found:[M/2+H] $]^{+}$).
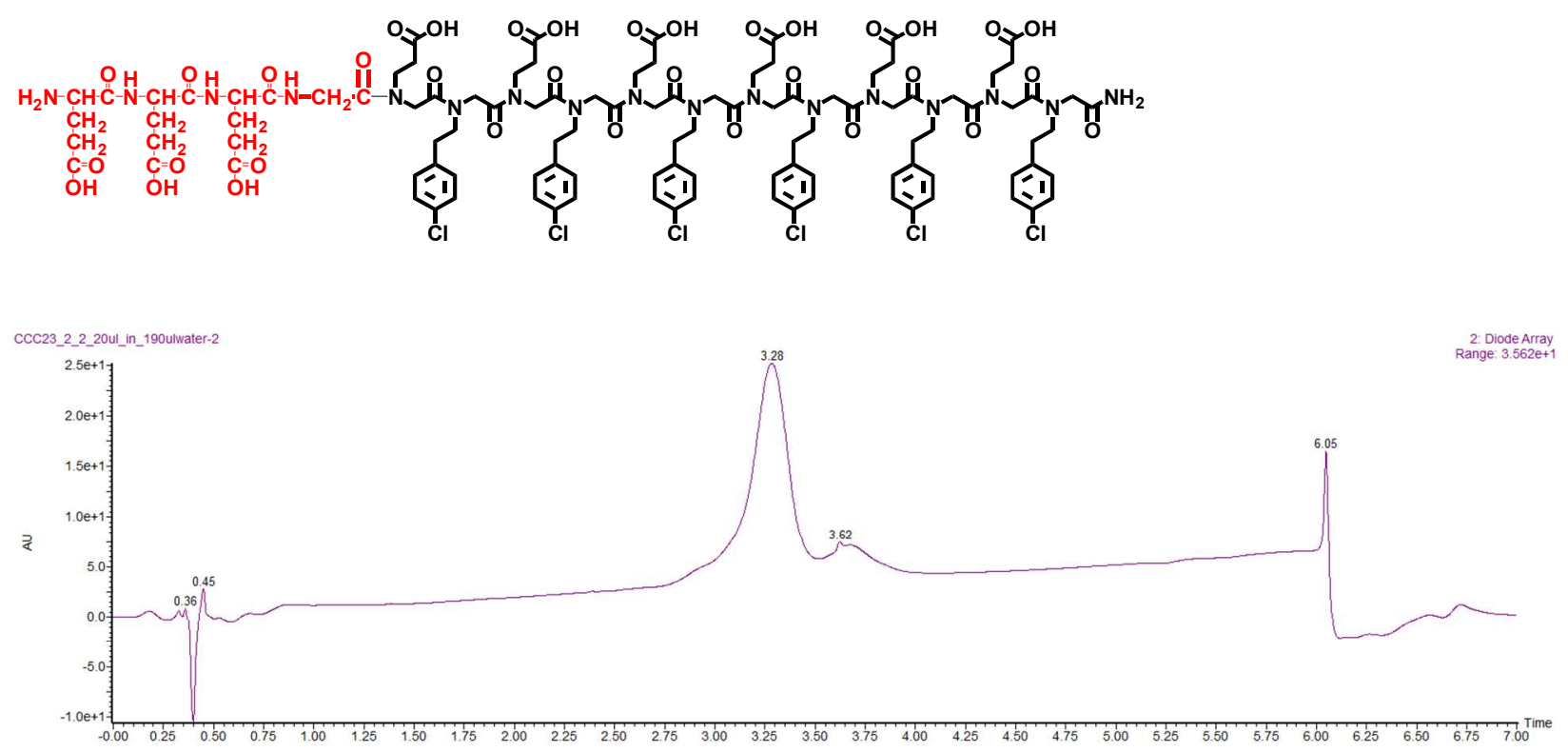

UPLC characterization of EEEG-Pep-1

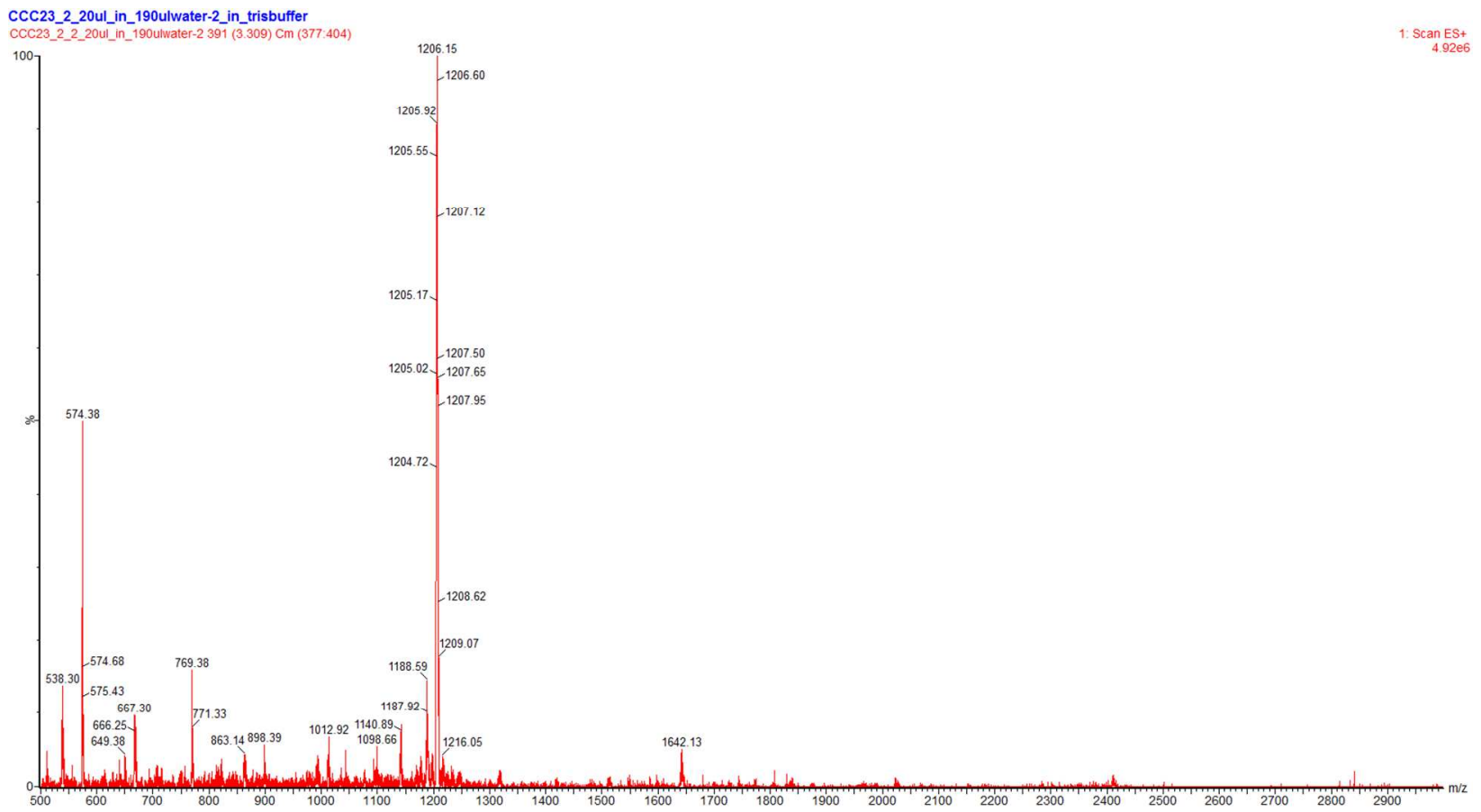

MS characterization of EEEG-Pep-1 
DGRG-Pep-1: 2351.0 (Molecular weight), (Found:[M+H] $]^{+}$), 1176.1 (Found:[M/2+H] $]^{+}$).

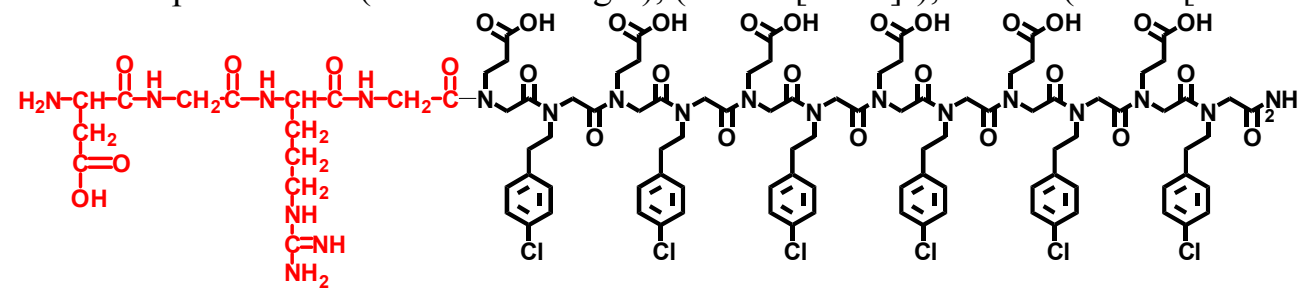

\section{CCC23_1_20ul_in_190ulwater}

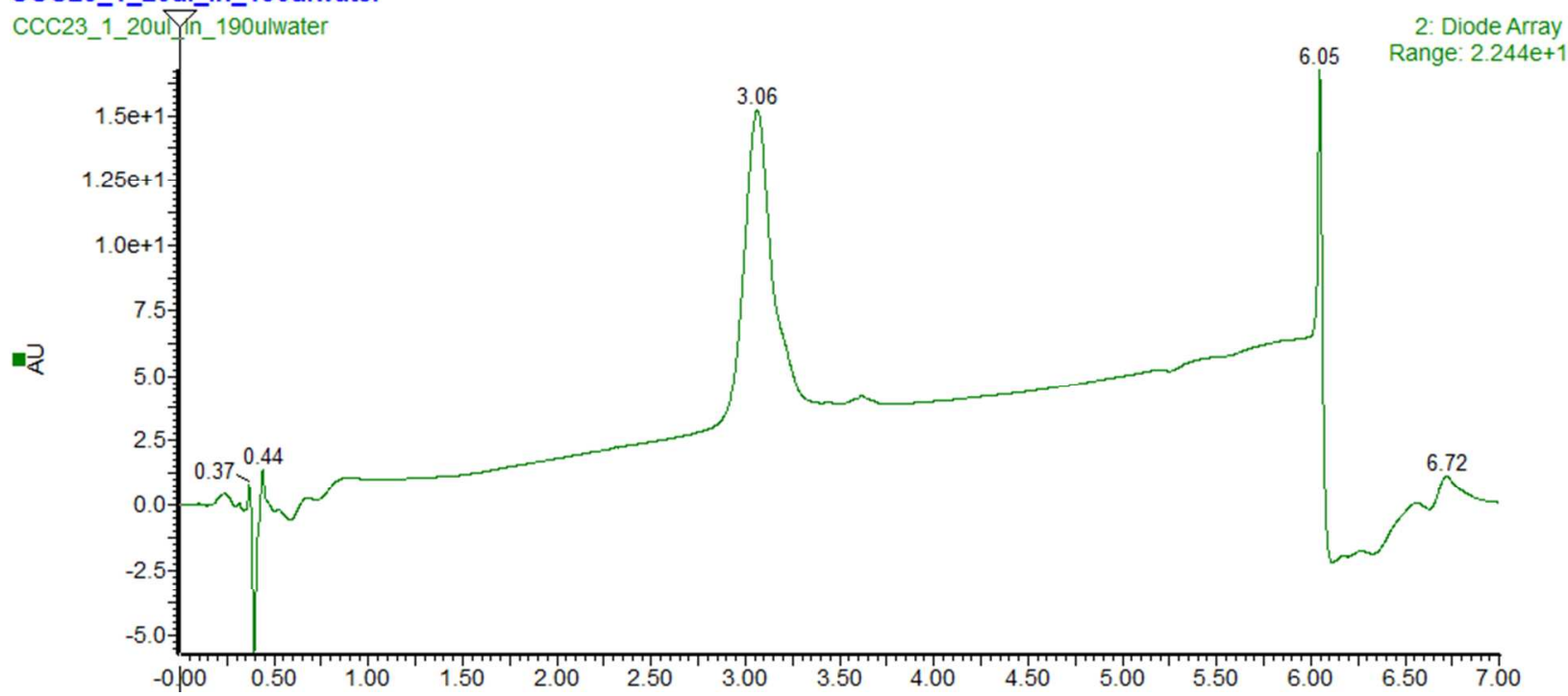

UPLC characterization of DGRG-Pep-1

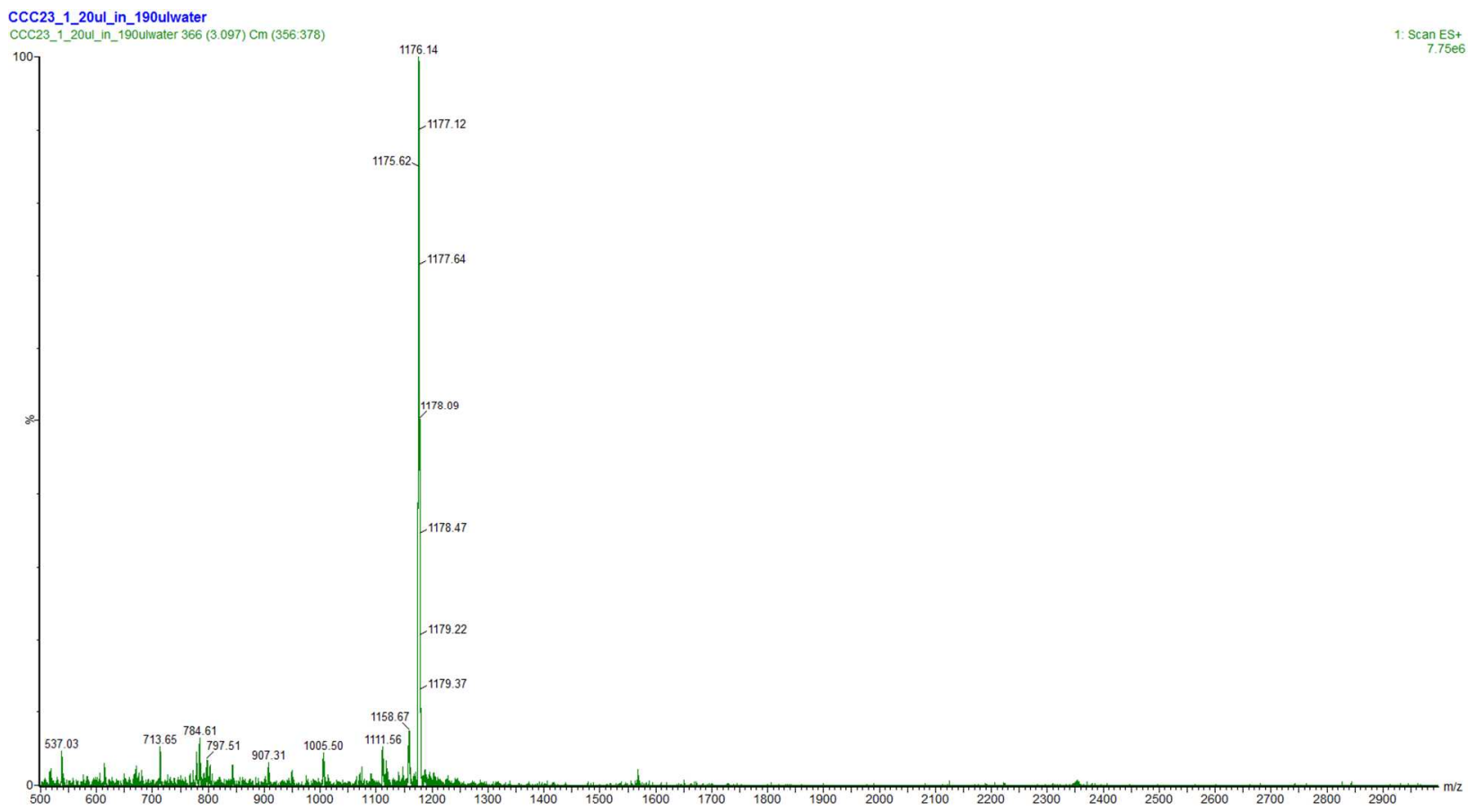

MS characterization of DGRG-Pep-1 


\section{Mica-directed assembly of peptoids}

$1.0 \mathrm{~mL}$ aqueous solution of $\mathrm{CaCl}_{2}(22 \mathrm{mM}), \mathrm{NaCl}(100 \mathrm{mM}), \mathrm{MgCl}_{2}(22 \mathrm{mM}), \mathrm{ZnCl}_{2}(22 \mathrm{mM}), \mathrm{CoCl}_{2}$ (100 mM), $\mathrm{FeCl}_{2}(22 \mathrm{mM})$, or $\mathrm{FeCl}_{3}(22 \mathrm{mM})$ was mixed with $1.0 \mathrm{~mL} 25 \mathrm{mM}$ Tris buffer $(\mathrm{pH}=8.0)$ in $4.0 \mathrm{~mL}$ glass vial, then $20 \mu \mathrm{L} 2.0 \mathrm{mM}$ peptoid stock solutions were added and well-mixed. The resultant solutions were incubated with freshly-cleaved mica and then left undisturbed at room temperature for assembly studies.

For self-assembly of Pep-1-Ca ${ }^{2+}$ complexes in the presence of $\mathrm{NaCl}$ aqueous solution: Aqueous solutions of $\mathrm{CaCl}_{2}(50 \mathrm{mM})$ and $\mathrm{NaCl}(1.0 \mathrm{M})$ were used to make a final assembling solution containing $25 \mathrm{mM}$ $\mathrm{CaCl}_{2}$ and $0.5 \mathrm{M} \mathrm{NaCl}$, while keeping the total solution volume and Pep-1 concentration same.

For characterizations of peptoid assemblies on mica surfaces, about 4 - 6 days later, mica surfaces from above incubated solutions were thoroughly washed with water and $\mathrm{N}_{2}$ dried before ex situ AFM imaging.

\section{AFM-based dynamic force spectroscopic study of peptoid-peptoid and peptoid-mica interactions} Microlever $\mathrm{Si}_{3} \mathrm{~N}_{4}$ AFM tips (Bruker, MSCT, CA) levers B, D or E were used in all experiments. The tip cleaning and cross-linking were done by using a similar method as described previously. ${ }^{3}$ Bare tips were first cleaned using a plasma cleaner for 20 seconds. These cleaned tips were immediately used for coating: they were coated with $4 \mathrm{~nm} \mathrm{Cr}$ followed by $\sim 20 \mathrm{~nm}$ Au by thermal evaporation. These coated probes were further plasma cleaned for $\sim 30$ seconds before they were immersed for 20 minutes in a anhydrous methanol solution containing $10 \mathrm{mM}$ of the heterobifunctional cross-linker LC-SPDP (Thermo Scientific) consisting of a pyridyl disulfide, which adsorbs to Au, and an N-hydroxysuccinimide (NHS) ester that reacts with the N-terminal amine or lysine of the peptide to form an amide bond. After rinsing well using anhydrous methanol, the tips were immersed in aqueous solution of Pep-1 $(\mathrm{pH}=\sim 8.0, \mathrm{NaOH}$ was used to adjust solution $\mathrm{pH}$ ) over night. Functionalized tips were rinsed well with water prior to use.

Force measurements between peptoid-modified tips and freshly-cleaved mica or pre-assembled Pep-1 nanoribbons on a mica surface were performed under water with a few drops of Tris buffer $(\mathrm{pH} 8.0,25$ $\mathrm{mM}$ ) solutions to adjust $\mathrm{pH}$. Measurements were made with the MFP3D Atomic Force Microscope (Asylum Research, Santa Barbara, CA). To account for any surface heterogeneity, a custom routine was used to randomly sample points on the surface to give a representative average. A constant approach velocity of $200 \mathrm{~nm} / \mathrm{s}$ was used for every pulling velocity studied. $\sim 200 \mathrm{pN}$ trigger forces were used to contact the surface and dwell for 0.99 second before pulling away. 


\section{S2. Supporting Figures}

a) With $\mathrm{NaCl}$

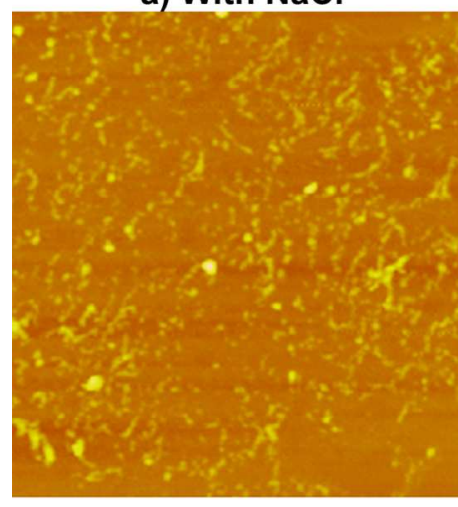

d) With $\mathrm{CoCl}_{2}$

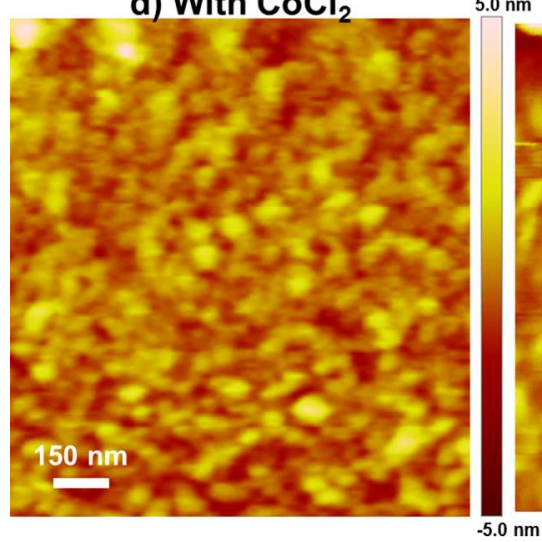

b) With $\mathrm{MgCl}_{2}$ $10 \mathrm{~nm}$

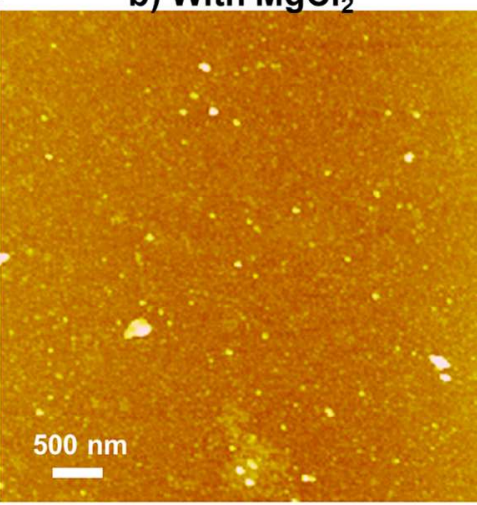

e) With $\mathrm{FeCl}_{2}$

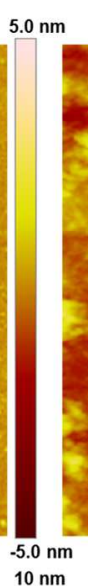

c) With $\mathrm{ZnCl}_{2}$
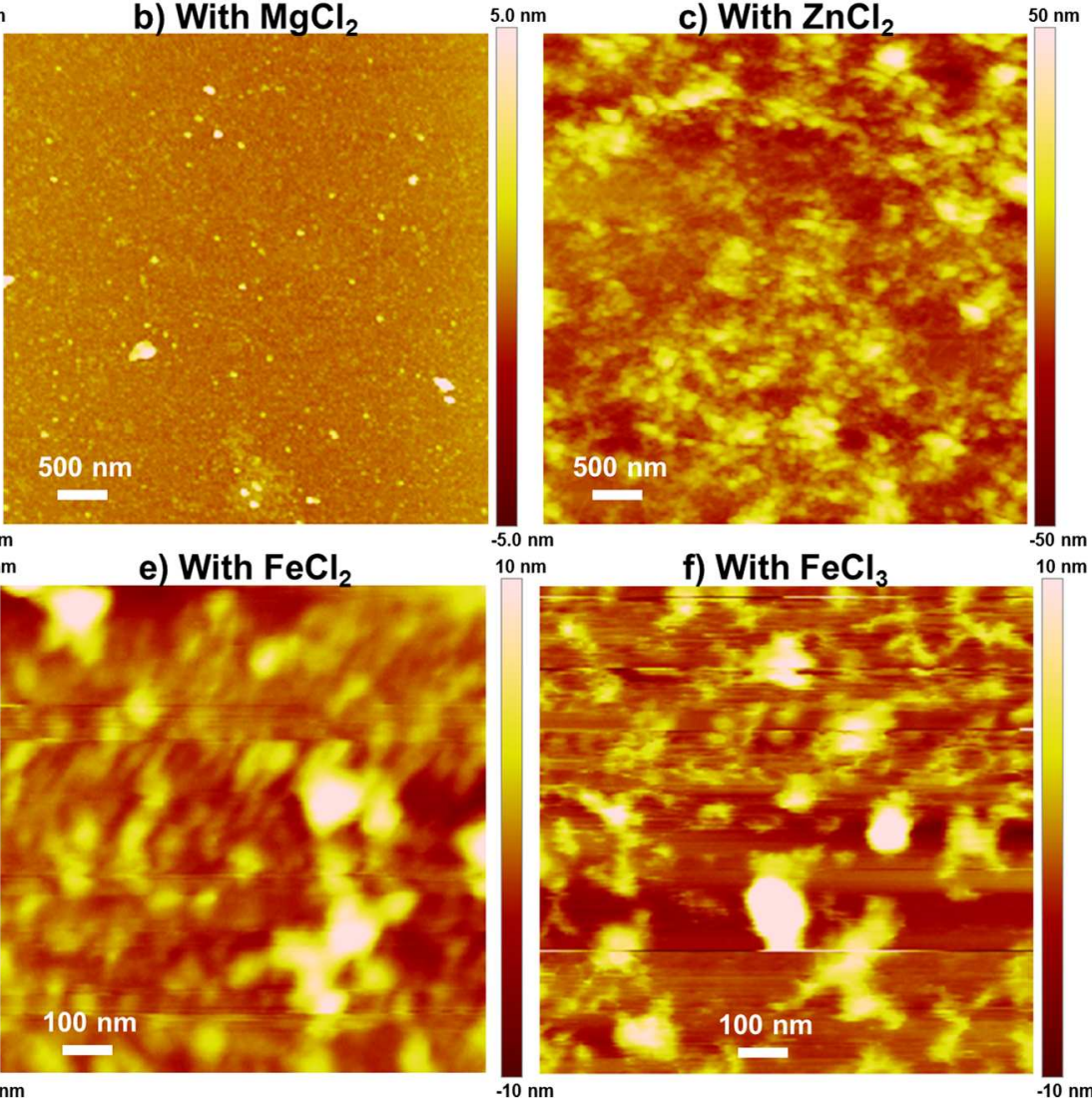

Figure S1. Assembly of Pep-1 on mica surfaces in the presence of other cations: a) $\mathrm{NaCl}$, b) $\mathrm{MgCl}_{2}$, c) $\mathrm{ZnCl}_{2}$, d) $\mathrm{CoCl}_{2}$, e) $\mathrm{FeCl}_{2}$ and f) $\mathrm{FeCl}_{3}$, which no ribbon structures were formed in the absence of $\mathrm{Ca}^{2+}$ cations; we reason that features of $\mathrm{Ca}^{2+}$ cations, which include the +2 charge, the size, and the coordination ability to carboxylate, are significant for the formation of hexagonally-patterned Pep-1 nanoribbon networks on mica surfaces.

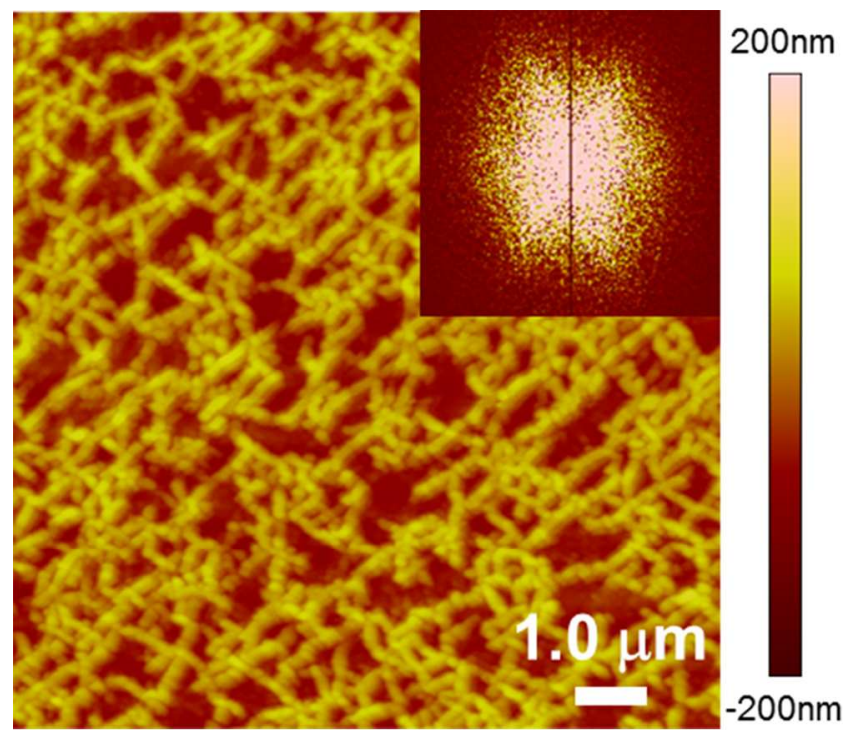

Figure S2. Pep-1 self-assembled into nanoribbon networks when even $0.5 \mathrm{M} \mathrm{NaCl}$ was present with 
$\mathrm{CaCl}_{2}(25 \mathrm{mM})$ in the solution used for assembly; the inset is a 2D Fourier transform of the main image showing the six-fold symmetry. Ex situ AFM data show that high ionic strength did not abolish nanoribbon formation, suggesting that hydrophobic interactions contribute significantly to nanoribbon stability.
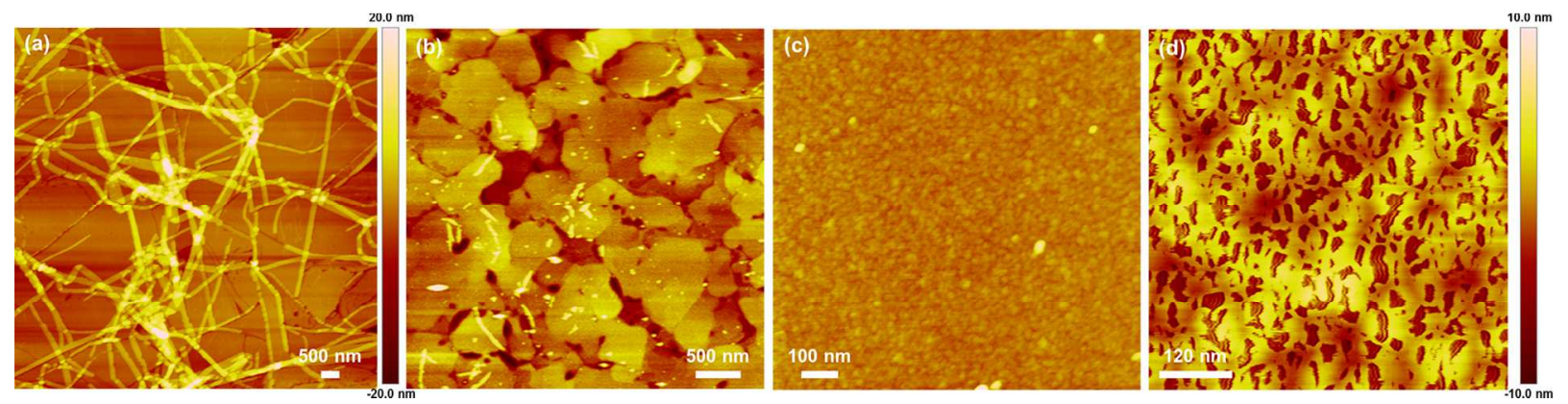

Figure S3. (a-d) Pep-1 self-assembly on four different surfaces: (a) on calcite (104) faces, (b) On Au(111) surface, (c) On silicon (111) surface, (d) On the carbon film of a TEM grid.

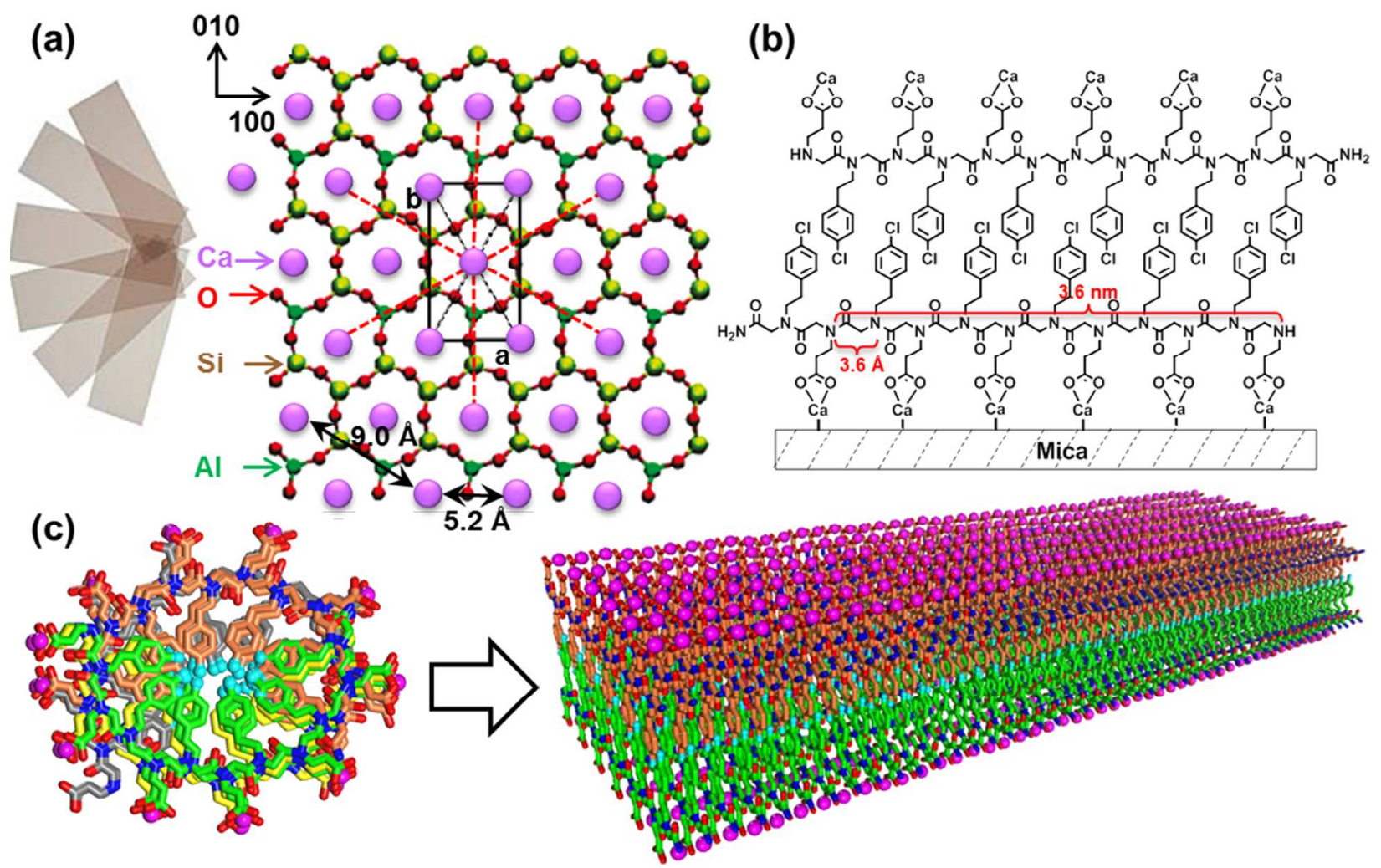

Figure S4. (a) The proposed model showing the locations of $\mathrm{Ca}^{2+}$ cations on the muscovite mica (001) plane, in which the $\mathrm{Ca}^{2+} \cdots \mathrm{Ca}^{2+}$ distance can be either 5.2 or $9.0 \AA \AA$; Peptoid ribbons are aligned along reddotted-line directions, and the carboxylate-carboxylate distances of Pep-1 match well with $\mathrm{Ca}^{2+} \cdots \mathrm{Ca}^{2+}$ distances $(3.6 \mathrm{~nm}=4 \times 9 \AA)$, while the flexibility of the peptoid backbone and sidechain $N-(2-$ carboxyethyl)glycines (Nce) groups as well as the potential mobility of absorbed $\mathrm{Ca}^{2+}$ cations at the muscovite mica surface ${ }^{4}$ make it possible for all other carboxylate groups to coordinate with $\mathrm{Ca}^{2+}$ cations; our unpublished high-resolution AFM data further confirmed this model. (b) The proposed model showing Pep-1 with deprotonated carboxylate groups bonded to mica surface through $\mathrm{Ca}^{2+}$ - carboxylate interactions; the $\mathrm{N} \cdots \mathrm{N}$ distance of Pep-1 backbone is $3.6 \AA$, and the carboxylate groups on both two ends 
have a distance of $3.6 \mathrm{~nm}$. (c) The proposed model showing the transformation of Pep-1 - $\mathrm{Ca}^{2+}$ discrete nanoparticles to nanoribbons on mica surfaces (nitrogen, blue; oxygen, red; calcium cation, purple; chloride, cyan).
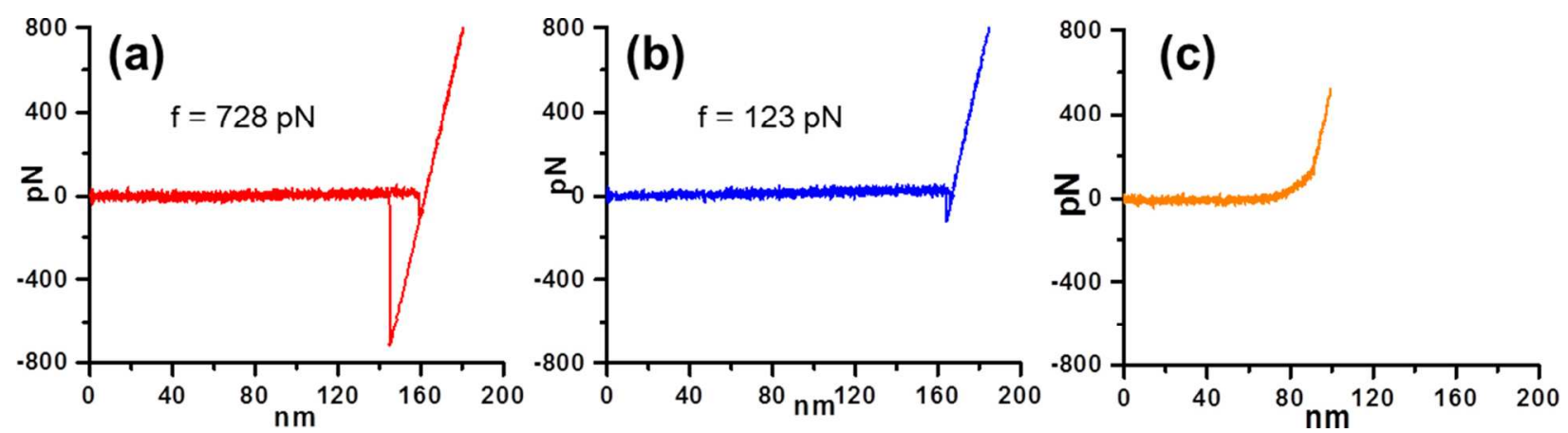

Figure S5. Representative force-distance curves at the retraction velocity $200 \mathrm{~nm} / \mathrm{s}$ showing Pep-1 functionalized AFM tips interact with mica (a), peptoid nanofibers (b) in the presence of $\mathrm{CaCl}_{2}$, or with mica in the absence of $\mathrm{CaCl}_{2}$ (c).
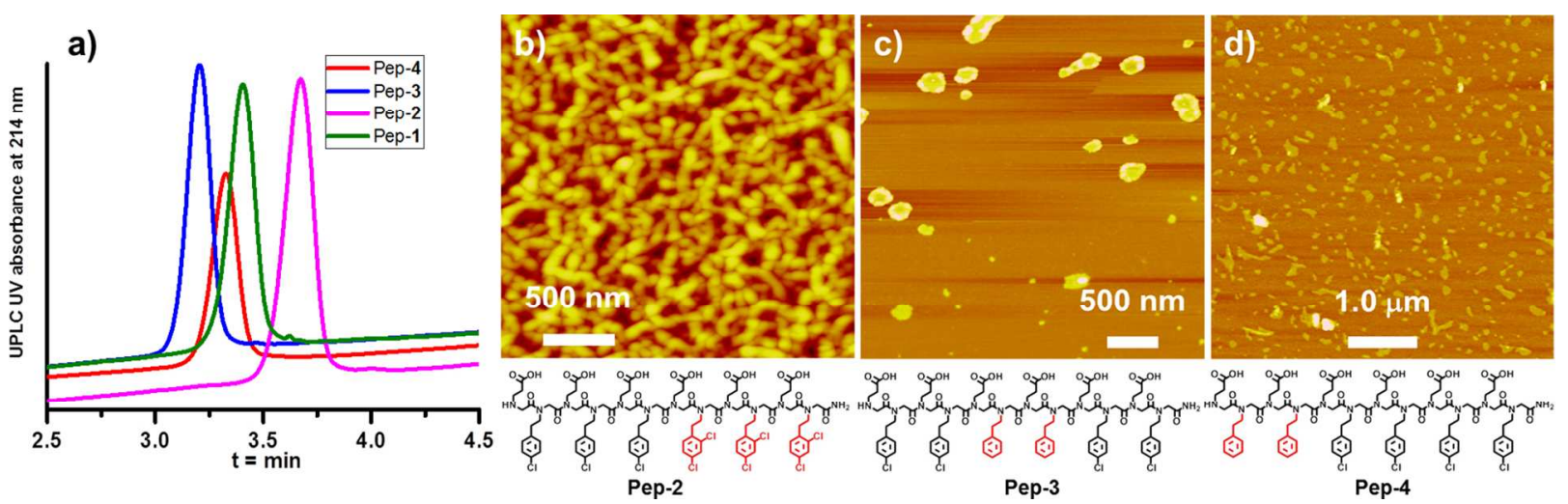

Figure S6. (a) Reverse phase UPLC data showing the relative hydrophobicities of Pep-1 - Pep-4, where longer retention time indicates higher hydrophobicity. (b-d) AFM images showing peptoid assembles from Pep-2 (a), Pep-3 (b) or Pep-4 (c) in the presence of aqueous $\mathrm{CaCl}_{2}$ solution. We reason that the resulting formation of randomly-packed nanoribbons of Pep-2 and no-assembly of Pep-2 and Pep-4 is due to the change of inter-peptoid hydrophobic interactions that are required to stabilize the nanoribbon formation.

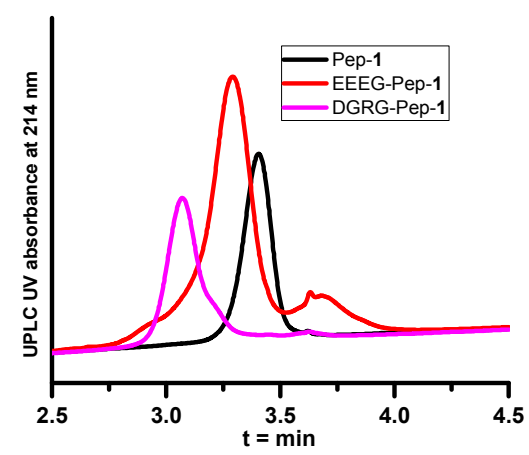

Figure S7. Reverse phase UPLC data showing the relative hydrophobicities of Pep-1, EEEG-Pep-1 and DGRG-Pep-1, where longer retention time indicates higher hydrophobicity. 


\section{S3. References}

(1) Zuckermann, R. N.; Kerr, J. M.; Kent, S. B. H.; Moos, W. H. Efficient Method for the Preparation of Peptoids Oligo(N-substituted Glycines) by Submonomer Solid-phase Synthesis. J. Am. Chem. Soc. 1992, 114, 10646-10647.

(2) Chen, C. L.; Qi, J. H.; Zuckermann, R. N.; DeYoreo, J. J. Engineered Biomimetic Polymers as Tunable Agents for Controlling CaCO3 Mineralization. J. Am. Chem. Soc. 2011, 133, 5214-5217.

(3) Friddle, R. W.; Battle, K.; Trubetskoy, V.; Tao, J.; Salter, E. A.; Moradian-Oldak, J.; De Yoreo, J. J.; Wierzbicki, A. Single-Molecule Determination of the Face-Specific Adsorption of Amelogenin's Cterminus on Hydroxyapatite. Angew. Chem., Int. Ed. 2011, 50, 7541-7545.

(4) Schlegel, M. L.; Nagy, K. L.; Fenter, P.; Cheng, L.; Sturchio, N. C.; Jacobsen, S. D. Cation Sorption on the Muscovite (001) Surface in Chloride Solutions using High-Resolution X-ray Reflectivity. Geochim. Cosmochim. Acta 2006, 70, 3549-3565. 